\title{
Perfil Multivariante de las mujeres empleadas en el Servicio Doméstico
}

\author{
$\mathrm{M}^{\mathrm{a}}$ Carmen PATINO AlONSO \\ Universidad de Salamanca \\ Departamento de Estadística \\ carpatino@usal.es \\ Marificación VICENTE GALINDO \\ Universidad de Salamanca \\ Departamento de Estadística \\ carpatino@usal.es \\ Ma Purificación GALINDO VILLARDÓN \\ Universidad de Salamanca \\ Departamento de Estadística \\ carpatino@usal.es
}

Recibido: 28-10-09

Aceptado: 4-10-10

\section{RESUMEN}

En este trabajo presentamos los resultados de un estudio realizado sobre las mujeres empleadas en el servicio doméstico, cuyo objetivo es conocer e identificar los perfiles socioeconómicos y laborales de las mujeres que se dedican a dicha actividad. En el análisis se han encontrado cuatro grupos bien diferenciados: uno formado por las mujeres empleadas de hogar regularizadas; otro constituido por mujeres inmigrantes y dos grupos más constituidos por mujeres de nacionalidad española, en situación laboral irregular diferenciados sobre todo en función de su estado civil.

Palabras clave: Servicio doméstico, Salamanca, biplot, análisis de correspondencias múltiples.

\section{Multivariate profile of the domestic workers}

\begin{abstract}
This paper reports the results of a study of women employed in the domestic help area that aims to identify the socioeconomic profiles of the women employed in this kind of activity. Four well differentiated groups were identified: one composed of regularized domestic-help workers working in the domestic area; another group formed by immigrant women, and a further two groups made up of women of Spanish nationality, in an irregular working situation, differentiated above all by their marital status.
\end{abstract}

Keywords: Domestic help, Salamanca, biplot, multiple correspondence analysis

\section{REFERENCIA NORMALIZADA}

Patino Alonso. MC., Vicente Galindo, $M^{a}$ P., y Galindo Villardón $M^{a}$ P. (2010). Perfil Multivariante de las Mujeres empleadas en el Servicio Doméstico. Cuadernos de Relaciones Laborales Vol. 29, núm. 2, p. 393-416 
SUMARIO: 1. Introducción. 1.1. Legislación laboral del servicio doméstico en España. 2. Objetivos. 3. Material y métodos. 4. Métodos estadísticos. 5. Descripción de la muestra. 5.1. Representatividad de la muestra. 5.2. Características sociodemográficas de las empleadas de hogar que conforman la muestra. 5.3. Características laborales. 6. Búsqueda de los perfiles de las mujeres empleadas de hogar, basada en información multivariante. 7. Demandas de las empleadas de hogar en situación laboral irregular para acceder al mercado de trabajo regular. 8. Medidas para mejorar el acceso al empleo regular. 9. Conclusiones. 10. Discusión. 11. Bibliografía. Notas finales

\section{Introducción}

En España, a principios del siglo XX, el menor nivel de industrialización y de desarrollo económico, en comparación con el entorno occidental, influyó en la participación laboral de la mujer. Dentro del sector terciario, el servicio doméstico constituía una de las actividades laborales tradicionalmente más desarrolladas por las mujeres, e indudablemente era la más importante desde un punto de vista cuantitativo, pues representaba el $72,6 \%$ del total de trabajadoras del sector servicios, sobrepasando el conjunto de empleos de la industria (González, 1982).

A principios del siglo XX, por encima de las ciudades castellanas, en las que los sirvientes formaban parte de manera consolidada del entramado social desde siglos atrás, sobresalía A Coruña, que no siguió con exactitud el patrón de las ciudades españolas, dado que a principios del siglo XX mostraba un porcentaje más elevado de población empleada en este subsector (Mirás, 2005).

El servicio doméstico desempeñó simultáneamente tres roles distintos. En primer lugar, la trabajadora satisfacía ciertas necesidades materiales, tales como la compra y elaboración de la comida, la limpieza y el mantenimiento general del hogar. En segundo lugar se hacía cargo del cuidado, educación y alimentación de los niños y personas mayores. Y finalmente, otra tarea era el desempeño de múltiples servicios que contribuían a mejorar la calidad de vida y el bienestar de sus beneficiarios (Sarasúa, 1983).

Después de la Segunda Guerra Mundial, estaba todavía en vigor el típico modelo de la mujer dedicada exclusivamente a su marido y al cuidado de los niños, una mujer que se dedicaría a las tareas domésticas y que no se preocuparía de lo que ocurriese fuera del hogar, dado que esto no se consideraba excesivamente femenino (Nielfa, 1993). Pero después de la Guerra Civil, debido a las tremendas dificultades socioeconómicas de la posguerra, se cambió el rol de la mujer en el mercado laboral incorporándose muchas de ellas como trabajadoras empleadas de hogar.

Cuando hablamos de "empleo del hogar" nos referimos a la relación laboral que se establece entre el titular de un hogar familiar (como empleador) y un trabajador (empleado) que presta servicios retribuidos en dicho hogar, realizando todo tipo de tareas domésticas.

El servicio doméstico engloba -total o parcialmente- aquellas actividades que habitualmente han realizado las mujeres inactivas o amas de casa, sin percibir remuneración a cambio y siendo socialmente consideradas carentes de valor. Por ello, las características especiales del servicio doméstico deben entenderse a partir 
de su condición de colectivo puente entre la economía doméstica y la economía monetaria (Colectivo IOÉ, 1996) ${ }^{1}$.

\section{1. Legislación laboral del servicio doméstico en España}

Hasta primeros del siglo XX, la actividad del servicio doméstico era meramente servil. Pero a partir de la llegada de la Democracia, las políticas laborales han intentado alejar el servicio doméstico de la servidumbre, tratando de equipararlo a la legislación laboral común. Con la Ley del Contrato de Trabajo, de 21 de noviembre de 1931, se reconoce por primera vez el carácter laboral de este sector. Más tarde, durante el franquismo se crean disposiciones sociales que incluyen a los servidores domésticos en el sistema de provisión social como a puesta en funcionamiento del Montepío Nacional del Servicio Doméstico, el 17 de marzo de 1959. Sin embargo, el empleo del hogar está contemplado como tal a partir del año 1985, ya que previamente el "servicio doméstico" estaba regulado por el Código Civil, lo que ya indica el carácter atípico de este tipo de empleo y la dificultad para asimilarlo a una relación laboral formal.

El artículo 2.1 b) del Estatuto de los Trabajadores considera relación laboral de carácter especial la del Servicio del Hogar Familiar, estableciéndose en la disposición adicional primera de la Ley 32/1984, de 2 de agosto, sobre modificación de determinados artículos de la Ley 8/1980, de 10 de marzo, del Estatuto de los Trabajadores, como el Gobierno, en el plazo máximo de doce meses, contados a partir de la entrada en vigor de la referenciada Ley, había de regular el régimen jurídico de las relaciones laborales de carácter especial previstas en el Estatuto de los Trabajadores (Ministerio de Trabajo y Seguridad social, Real Decreto 1424/1985 de 1 de Agosto - BOE 13 agosto 1985, núm. 193). La normativa que regula el servicio doméstico es una norma gubernativa, no se trata de un convenio colectivo.

Dicho Decreto incluye todas las actividades de ámbito doméstico -como el cuidado de la casa y la atención a los miembros de la familia o de las personas que convivan en el domicilio-. También comprende otras labores como la jardinería o la conducción, siempre que estén relacionadas directamente con las tareas domésticas. En este trabajo hemos incluido, además de las tareas y mantenimiento del hogar, otras como el cuidado de menores, de personas ancianas, personas enfermas y discapacitados. Además hemos incluido a las mujeres que trabajan en limpieza de oficinas, dada la similitud e irregularidad laboral en ambas tareas.

Dicha legislación ha permanecido prácticamente invariable desde su publicación, a pesar de que se trata de una tipo de relación laboral que afecta a un gran número de trabajadores en España.

Entre las principales cuestiones que podemos destacar a efectos de relaciones laborales son: la normativa exime a los empleadores de realizar un contrato laboral

${ }^{1}$ Colectivo IOÉ: Equipo de investigación social radicado en Madrid (España), que desarrolla investigaciones empíricas, cursos y seminarios de formación 
por escrito, con lo que se facilita la indeterminación de los periodos de descansos semanales; estos trabajadores cuentan con una indemnización de siete días por año trabajado con un máximo de seis mensualidades, mientras que los demás asalariados disfrutan de 33 días por año cumplido y con un límite de 24 sueldos; el salario mínimo a percibir es el salario mínimo interprofesional fijado por el Gobierno (el SMI para 2009 es de 8.736 euros -12 pagas mensuales y 2 medias pagas- ); el empleado de hogar tiene derecho a dos pagas extras de carácter semestral equivalente a 15 días de salario cada una de ellas, por cada tres años de antigüedad se incrementará el salario en un 3\% con un máximo de 5 trienios (estas retribuciones sólo empiezan a contar a partir de 1986); la jornada será de 40 horas semanales como máximo y 9 horas diarias y no se autoriza a realizar más de 80 horas extraordinarias al año (con respecto a esto, la normativa prevé que se establezca disponibilidad en el hogar, no computables como horas extraordinarias).

Existe un Régimen Especial de las Empleadas de Hogar con una cobertura claramente discriminatoria al Régimen General. Esto conduce a que exista un elevado índice de economía sumergida ("irregular") que afecta a la mayoría de las trabajadoras del servicio doméstico (alrededor del 60\%); esta cifra casi duplica la existente entre el conjunto de mujeres y es tres veces superior a la que se registra entre los hombres ocupados (Colectivo IOÉ, 1990).

El obligado al pago de las cotizaciones es el empleador, siempre y cuando se trate de una situación en la que el trabajador ha sido contratado bajo la modalidad de empleado de hogar fijo (el calificativo fijo está relacionado con el número de horas prestadas al mes en el domicilio, no tiene nada que ver con a duración del contrato), si trabajan para más de un empleador deben asumir el pago de su propia cuota de afiliación, lo que los convertiría en una especie de trabajadores autónomos, cuota que ronda los 146 euros mensuales (esto explica que muchos de los empleados domésticos, en lugar de tener varios trabajos, prefieran trabajar como internos para tener el beneficio de ser dados de alta por el empleador, tal como exige la ley). Este régimen no permite que se den de alta las personas que trabajen menos de 72 horas mensuales, pero también, se dan muchos casos en los que los empleados prefieren trabajar en negro y ahorrar el dinero que deberían invertir en la Seguridad Social, de esta manera, no tienen contrato de trabajo y no gozan de los beneficios de estar adscritos a la Seguridad Social. La familia está obligada a pagar a la Seguridad Social si precisa el servicio doméstico más de 20 horas semanales, pero la norma sólo se aplica si hay un único empleador, cuando hay varios, el pago corresponde a la trabajadora. La principal laguna de este sistema es qué ocurre con quienes desempeñan su labor menos de 20 horas a la semana.

El empleado de hogar no tiene derecho a desempleo ni a realizar retenciones de IRPF en los pagos al trabajador, en caso de enfermedad no cobrará hasta pasados 29 días de baja por enfermedad (mientras dure la baja de enfermedad, el empleador podrá dar de baja en la Seguridad Social al empleado).

La prestación la cobrará el empleado directamente del INSS. La inexistencia de prestación por desempleo y lo que concierne a la extinción de la relación laboral, 
son las cuestiones más problemáticas por la situación de desprotección social que suponen para el trabajador.

Las peculiaridades de este régimen se justifican por el hecho de que el servicio doméstico se es una prestación de trabajo que se realiza en el hogar, lo que hace necesario una mutua confianza entre las partes y flexibilidad (Quesada, 1991)

Dado que la característica principal del trabajador del servicio doméstico es la convivencia con el empleador, las normativas que se han desarrollado en España tratan de preservar su intimidad (Luján, 2000).

Tras largas negociaciones entre Gobierno y sindicatos, para el año 2008, el Gobierno propuso un borrador con una reforma sobre la normativa del REEH donde los puntos fundamentales son que las empleadas de hogar deberán firmar un contrato con su empleador debiendo ser comunicados a los servicios públicos de empleo, recibirán una prestación cuando estén de baja por accidente laboral y se les reconoce el derecho a paro aunque de momento se aplaza su regulación. En cuanto a la obligación de cotizar las condiciones no varían prácticamente con respecto al antiguo sistema.

Según publica el diario "Tribuna de Salamanca" con fecha 6.11.2008 (Gómez, 2008), sólo una de cada tres empleadas del hogar cotiza a la Seguridad Social, esto es, de las 4.500 trabajadoras que desempeñan este oficio, solamente 1.500 están dadas de alta, siendo el 66 por ciento de las empleadas de hogar inmigrantes.

Sin duda alguna, el servicio doméstico es una actividad eminentemente femeni$\mathrm{na}^{2}$, con una elevada incidencia de empleo irregular y de situaciones encuadradas en la denominada "economía informal"3 como por ejemplo, las empleadas de hogar que no generan obligación de cotizar.

Este sector requiere una reforma del sistema laboral donde el colectivo goce de un trabajo decente, sinónimo de trabajo productivo, en el cual se protejan los derechos y suponga ingresos adecuados con una protección social apropiada, porque el trabajo es una actividad con funciones positivas como la económica (Ruiz Quintanilla y Wilpert, 1988), pero también con funciones negativas como son las características del puesto así como las condiciones ambientales y sociales en las que se desempeña (Giddens, 1989).

En un contexto de crisis económica como el que se está actualmente viviendo en el país y en el cual España ha experimentado uno de los procesos migratorios más intensos de Europa, siendo el servicio doméstico según la Encuesta de Población Activa la actividad laboral con mayor nivel de extranjeros, conlleva a una mayor

${ }^{2}$ Según la Encuesta de Población Activa (EPA) elaborada por el Instituto Nacional de Estadística (INE) durante los últimos años los hombres representan alrededor del $12 \%$ de las personas ocupadas en el sector.

${ }^{3}$ Economía informal: actividades productivas no retribuidas. Es la producción que no es objeto de intercambio en el mercado y no cuenta con el dinero como instrumento de cambio. 
movilidad de los asalariados, y en muchos casos, además, a un fuerte deterioro de las condiciones laborales (Castillo, 2005).

En este estudio nos hemos centrado en el colectivo de las mujeres, por el importante peso que éstas tienen en la actividad del servicio doméstico y por la situación de irregularidad laboral tan acusada que les afecta en dicho sector. Para abordar la cuantificación del fenómeno y poder ofrecer de forma ilustrativa la realidad actual de estas trabajadoras, dada la ausencia de información ofrecida por fuentes estadísticas oficiales, el único procedimiento viable para aproximarnos al conocimiento del problema requiere una forma de estimación "directo", es decir, un procedimiento basado en entrevistas realizadas a las mujeres implicadas, para ello hemos seleccionado una muestra de mujeres empleadas en el servicio doméstico. El espacio geográfico de la investigación es la provincia de Salamanca.

En el siglo pasado, en Salamanca se produjo el tránsito de una economía agraria tradicional a una de mercado, pero esas transformaciones de la economía rural no han sido capaces de crear puestos de trabajo específicos para las mujeres, con lo cual muchas de ellas han tenido que emigrar para poder desempeñar trabajos remunerados en los núcleos urbanos, siendo el sector del servicio doméstico donde encuentran su principal salida laboral y el más numeroso para el sexo femenino. A pesar de estos cambios, la incorporación de las mujeres al mercado laboral de Salamanca sigue siendo inferior a la que presentan otras provincias debido al menor dinamismo y capacidad de economía de la provincia para generar empleo.

A principios de siglo, Salamanca era la sexta provincia de acuerdo al porcentaje de población femenina empleada en el servicio doméstico a nivel nacional (tan sólo era superada por algunas ciudades grandes como San Sebastián, Bilbao o Madrid) y la segunda en la Comunidad de Castilla y León (superada por Ávila) (Mirás, 2005). Esto revela que la presencia del sector del servicio doméstico en la provincia era ya elevada por entonces, por ello nos ha parecido indicativo el lugar para desarrollar el trabajo de campo. El número total de encuestas analizadas son 878 y mediante las cuales tratamos de conocer de forma general el perfil y la problemática de las mujeres ocupadas en dicha actividad y el efecto que tiene la situación de irregularidad laboral de estas trabajadoras en la economía.

\section{Objetivos}

En este trabajo tratamos de investigar en profundidad la situación de las empleadas de hogar, dado el interés que tiene este colectivo en el contexto del trabajo irregular.

Nos planteamos los siguientes objetivos: identificar los perfiles de las mujeres salmantinas empleadas en el servicio doméstico: edad, nivel educativo, cualificación profesional, nivel de ingresos etc.; detectar su auto-imagen, así como las principales actitudes, opiniones y expectativas, respecto a su situación laboral; captar los estereotipos sociales, referidos al servicio doméstico; estimar el porcentaje 
de empleadas de hogar salmantinas en situación regular e irregular y estimar el peso que tienen, en el servicio doméstico, las inmigrantes.

\section{Material y métodos}

La unidad estadística objeto de investigación son mujeres que se dedican a la actividad del servicio doméstico, que trabajen en la provincia de Salamanca, con edad comprendida entre 16 y 65 años, empleadas de manera regular o irregular.

Para configurar las dimensiones y los ítems que conforman el cuestionario se han realizado entrevistas en profundidad y grupos de discusión, con el fin de obtener una visión global del fenómeno objeto de estudio desde distintas perspectivas: la opinión de las personas expertas sobre irregularidad laboral femenina: Red Mujer Empleo de la Junta de Castilla y León, Área de Bienestar Social, Cruz Roja, Asociaciones de Mujeres, Sindicatos, Investigadores de la Universidad de Salamanca y la opinión de las mujeres trabajadoras sobre su situación laboral.

El Cuestionario consta de 50 preguntas que recogen Características Sociodemográficas, Formación y Cualificación, Situación laboral y trabajo, Consecuencias de la situación de irregularidad sobre la vida laboral y personal, Demandas y Medidas.

La muestra se recogió de la siguiente manera: en una primera etapa se clasificaron los municipios en función de las características socio-demográficas que constan en el Anuario Social de España 2004, publicado por la Fundación La Caixa, el cual ofrece una recopilación de Indicadores Sociales para municipios de más de 1000 habitantes. Ver tabla 1. Se utilizó la técnica exploratoria multivariante, HJ-Biplot (Galindo, 1986). Este análisis busca direcciones principales de inercia, como un análisis de Componentes Principales, pero permite la representación simultánea de los municipios y las variables sociodemográficas, sobre el espacio de máxima inercia, con máxima calidad de representación.

Los municipios se representan como puntos en un espacio vectorial de dimensión dos, en el cual es posible interpretar la proximidad entre los puntos que representan a los municipios en términos de similaridad; es decir, si dos municipios están próximos en el gráfico Biplot, eso significa que tienen características sociodemográficas similares. Las longitudes y los ángulos de los vectores que representan a las variables, se interpretan en términos de variabilidad y covariabilidad respectivamente. El HJ-Biplot proporcionó los siguientes grupos con características bien diferenciadas, en relación a las variables consideradas:

Grupo 1: Salamanca capital. Grupo 2: Aldeadávila de la Ribera, Cantalapiedra, Cantalpino, Linares de Riofrío, Lumbrales, Macotera, SanctiSpiritus y Villarino de los Aires. Grupo 3: Alba de Tormes, La Alberca, Béjar, Calvarrasa de Abajo, Ciudad Rodrigo, La Fuente de San Esteban, Guijuelo, Peñaranda de Bracamonte, Villoria y Vitigudino. Grupo 4: Cabrerizos, Carbajosa de la sagrada, Santa Marta de Tormes, Terradillos. Grupo 5: Pueblos con menos de 1.000 habitantes. 
En una segunda etapa, dadas las características del estudio, se realizó un muestreo en bola de nieve, en cada una de estos grupos resultantes del Biplot. La muestra está compuesta de 878 mujeres, 544 mujeres en situación irregular y 334 mujeres en situación regular.

El tamaño de muestra en cada Grupo de municipios se eligió de manera proporcional al tamaño del Grupo, incrementándose el número de encuestas en la capital por ser el lugar donde se produce la mayor concentración de mujeres empleadas de hogar.

Tabla 1. Indicadores sociodemogràficos utilizados en el estudio multivariante para la caracterización de los estratos

\begin{tabular}{|l|l|}
\hline HOMB & Porcentaje de la población de HOMBres en cada uno de los municipios \\
\hline MUJE & Porcentaje de la población de MUJEres en cada uno de los municipios \\
\hline EMP & Edad Media Poblacional de cada municipio \\
\hline EMM & Edad Media de las Mujeres en cada municipio \\
\hline IDEP & Índice de DEPendencia \\
\hline PC & Población Compacta \\
\hline NMMU & Índice de Nacidos en el Mismo MUnicipio \\
\hline NMPR & Índice de Nacidos en otro Municipio de la misma PRovincia \\
\hline NMCO & Índice porcentual de Nacidos en otro Municipio de \\
& la misma COmunidad Autónoma \\
\hline NOCO & Nacidos en Otra COmunidad Autónoma \\
\hline NEXT & Índice de Nacidos en el EXTranjero \\
\hline TANA & Tasa de Natalidad \\
\hline TAFE & Tasa de Fecundidad. \\
\hline TAMO & Tasa de Mortalidad \\
\hline CRVE & Tasa de CRecimiento Vegetativo \\
\hline TANU & TAsa de NUpcialidad \\
\hline TAMI & TAsa de MIgración \\
\hline PEPA & Índice de la estructura de la Población en Edad Potencialmente Activa \\
\hline PARO & Tasa de PARO \\
\hline INF & Índice de INFancia \\
\hline IJUV & Índice de JUVentud \\
\hline IDIN & Índice de Dependencia INfantil \\
\hline IVEJ & Índice de VEJez \\
\hline ISEN & Índice de SENectud \\
\hline IENV & Índice de ENVejecimiento \\
\hline IDEM & Índice de DEpendencia de personas Mayores \\
\hline REPM & REsidencias Por cada Mil ancianos \\
\hline TCE & Total Centros Escolares \\
\hline
\end{tabular}




\begin{tabular}{|l|l|}
\hline ANF & ANalFabetos \\
\hline PR & Número de Plazas de Residencias destinadas a las personas mayores \\
\hline
\end{tabular}

\section{Métodos estadísticos}

Para buscar los diferentes perfiles de empleadas de hogar, se realizó un análisis de Homogeneidad (HOMALS), -también conocido como Análisis Factorial de Correspondencias Múltiples- procedimiento multivariante no lineal que se computa utilizando mínimos cuadrados alternados (Gifi, 1990). De esta forma las mujeres empleadas de hogar que seleccionan la misma categoría de respuesta, para una variable, aparecen próximas en el gráfico multivariante, proyectado desde el hiperespacio, mientras que aquellas que eligen categorías diferentes aparecen distantes en el gráfico. Cada empleada de hogar aparece próxima al conjunto de categorías seleccionadas por ella, al responder al cuestionario. Partiendo de las coordenadas de las mujeres en el espacio Biplot, y aplicando un análisis de Conglomerados de kmedias, se encontraron los perfiles multivariantes de las trabajadoras en el servicio doméstico. La caracterización de los perfiles se llevó a cabo utilizando el test Chi cuadrado.

\section{Descripción de la muestra}

\subsection{Representatividad de la muestra}

La encuesta ha sido diseñada para representar estadísticamente al conjunto de la provincia de Salamanca, distribuyéndolas según el peso estimado que en las diferentes zonas tiene el empleo en el servicio doméstico.

De los 362 municipios que constituyen la provincia de Salamanca hemos muestreado 68 , aproximadamente un $20 \%$ del total.

La distribución encontrada en relación con el tipo de municipio es la siguiente ${ }^{4}$ : en la muestra considerada un 3,8\% pertenecen a municipios que tienen menos de 500 habitantes $(0,3 \%$ de las encuestas a pueblos de menos de 100 habitantes y $3,5 \%$ entre 100 y 500 habitantes) - ruralidad estricta -; el 7,5\% de la muestra pertenece a municipios de menos de 2.000 habitantes - ruralidad intermedia -; 7,9\% tienen

${ }^{4}$ Según García (1998), se clasifican como rurales los municipios con menos de 10.000 habitantes y los municipios de más de 10.000 habitantes como no rurales. Sin embargo, distingue diferentes niveles de ruralidad: ruralidad laxa, que se corresponde con umbrales de población de 2.000 a 10.000 habitantes; ruralidad media que afecta a los municipios de 500 a 2.000 habitantes, y ruralidad estricta que es la propia de los municipios con menos de 500 habitantes. 
entre 2.000 y 10.000 habitantes - ruralidad laxa -; y un $80,9 \%$ corresponde a municipios con más de 10.000 habitantes - urbana.

En la provincia de Salamanca, los municipios no rurales (urbanos), es decir, los que tienen más de 10.000 habitantes son: la capital, Santa Marta de Tormes, Béjar y Ciudad Rodrigo. El resto siguiendo la clasificación de García (1998) son municipios rurales.

Los porcentajes más altos, tanto en el caso de las mujeres que trabajan en condiciones laborales regulares como no regulares, se concentran en la zona urbana $(79 \%$ y $82 \%$ respectivamente). Un $62,8 \%$ de las trabajadoras que lo hacen en zona urbana lo desarrollan en condiciones laborales irregulares y un 37,2\% de manera regularizada. Observamos como estos porcentajes son relativamente próximos para el caso de las zonas rurales (58,3\% y 41,7\% respectivamente). En el 51,5\% de los municipios con ruralidad intermedia (La Alberca, Candelario, Villoria, etc.), las trabajadoras en el servicio doméstico desarrollan su trabajo en situación laboral regular, presentando en el resto de municipios porcentajes más bajos. Ver tabla 2.

Tabla 2. Distribución de las empleadas de hogar según situación laboral atendiendo al tipo de municipio

\begin{tabular}{|l|l|c|c|c|c|c|c|}
\hline \multicolumn{2}{|l|}{} & $\begin{array}{c}\text { Rural } \\
\text { Total }\end{array}$ & $\begin{array}{c}\text { Rural } \\
\text { Estricta }\end{array}$ & $\begin{array}{c}\text { Rural } \\
\text { Intermedia }\end{array}$ & $\begin{array}{c}\text { Rural } \\
\text { Laxa }\end{array}$ & Urbana & TOTAL \\
\hline REGUL & Recuento & 70 & 15 & 34 & 21 & 264 & 334 \\
\hline & $\begin{array}{l}\text { \%Tipo } \\
\text { de Municipio }\end{array}$ & $\mathbf{4 1 , 7 \%}$ & $45,5 \%$ & $\mathbf{5 1 , 5 \%}$ & $30,4 \%$ & $\mathbf{3 7 , 2 \%}$ & $38,0 \%$ \\
\hline IRREG & Recuento & 98 & 18 & 32 & 48 & 446 & 544 \\
\hline & $\begin{array}{l}\text { \%Tipo } \\
\text { de Municipio }\end{array}$ & $\mathbf{5 8 , 3 \%}$ & $54,5 \%$ & $\mathbf{4 8 , 5 \%}$ & $69,6 \%$ & $\mathbf{6 2 , 8 \%}$ & $62,0 \%$ \\
\hline TOTAL & Recuento & 33 & 33 & 66 & 69 & 710 & 878 \\
\hline
\end{tabular}

\subsection{Características socioedemográficas de las empleadas de hogar que con- forman la muestra}

La muestra objeto de estudio está compuesta por 878 trabajadoras empleadas en el sector del servicio doméstico, de las cuales el $62 \%$ desarrollan su actividad en un marco laboral irregular (no cotizan debidamente a la Seguridad Social) ${ }^{5}$ y un $38 \%$ están regularizadas.

En muchos países europeos, las trabajadoras empleadas en el servicio doméstico, nacionales y extranjeras, no están registradas en el sistema de la Seguridad Social (Destremau, 2002).

${ }^{5}$ Este análisis al referirse al término de "empleo irregular" se centra en comportamientos que suponen una merma en la recaudación de las cotizaciones sociales. 
El 28\% de las mujeres de la muestra son de nacionalidad extranjera, la mayoría procedentes de Latinoamérica encontrándose el $71,7 \%$ de ellas en situación laboral irregular.

En este sector, el 46,6\% de las trabajadoras en situación laboral irregular tienen una edad comprendida entre 30 y 45 años y el $32,5 \%$ tiene más de 45 años.

El $68 \%$ de las mujeres que trabajan en el servicio doméstico tienen estudios primarios o carecen de algún tipo de estudio. Este menor nivel académico está relacionado con la mayor edad media que tienen las mujeres que trabajan en el sector del servicio doméstico.

En cuanto al estado civil, hay un mayor porcentaje de mujeres casadas o parejas de hecho $(62 \%)$, situación relacionada también con la mayor edad media de las mujeres que trabajan en dicho sector.

Estos datos ponen claramente de manifiesto como es un sector donde existe un elevado índice de economía sumergida, la cual representa en España más del 20\% del producto interior bruto (PIB). En el trabajo "La presencia de las mujeres en el empleo irregular" realizado por el Cabo y col. (2005) respecto a la nacionalidad destaca la mayor presencia de trabajadoras extranjeras entre las empleadas de hogar (19\%), siendo tan sólo un 3\% de las regularizadas inmigrantes. Una de las principales características de la composición de los actuales flujos migratorios que llegan a España es su tendencia a la femenización y la latinoamericanización (Izquierdo y col., 2003).

\subsection{Características laborales}

En este apartado se analiza la trayectoria laboral, es decir, la experiencia laboral, la antigüedad, y las vías de acceso a su actual empleo de las mujeres que trabajan como empleadas del hogar. Además siempre que la muestra lo permite, se estudian las diferencias que se dan entre las mujeres de nacionalidad española y extranjera y las diferencias que se producen entre las mujeres en situación laboral regular y las que están en situación laboral irregular.

En lo que se refiere a la experiencia laboral, entre las mujeres que trabajan en el servicio doméstico (tanto en situación laboral regular e irregular) aproximadamente el $81 \%$ han trabajado con anterioridad al empleo actual. De lo que se puede concluir por tanto, que hay una gran movilidad laboral entre las empleadas de hogar, mostrando una gran inestabilidad en su trabajo.

En cuanto a la antigüedad en el servicio doméstico, en ambos casos tanto en el caso de las mujeres que se encuentran en situación laboral irregular como las regularizadas, el porcentaje de mujeres con más de tres años en el sector es elevado, para las primeras asciende a un $43,2 \%$ y en el caso de las mujeres en situación laboral regular a un 53,3\%.

Teniendo en cuenta la nacionalidad de las mujeres, apreciamos que tan sólo un $22,8 \%$ de las mujeres extranjeras que trabajan en el servicio doméstico, en situación laboral irregular, lleva más de tres años, siendo lo habitual que lleven entre uno y tres años (46\%). En el caso de las mujeres españolas el $57 \%$ de las mismas tiene una antigüedad en el sector superior a los 3 años. Estas diferencias se explican 
porque para las mujeres extranjeras el servicio doméstico supone en muchas ocasiones una vía de entrada al mercado laboral, siendo sólo una etapa transitoria mientras encuentran un empleo más estable o mejor pagado.

Las mujeres inmigrantes ilegales en particular se encuentran en una posición vulnerable: sin posibilidades de cualquier desarrollo profesional, con derechos muy limitados para negociar el salario o las horas de trabajo y sin ayuda legal para resolver problemas profesionales.

Las mujeres españolas que trabajan en el servicio doméstico son la mayoría mujeres casadas, con hijos y este trabajo les permite compatibilizar la obtención de ingresos con la atención de su familia.

Analizando las vías de búsqueda de empleo, el camino más utilizado para la obtención del empleo es a través de la familia o amigos. El 55,8\% de las mujeres en situación laboral irregular encontraron el trabajo a través de amigos o familiares; el $50 \%$ de las empleadas en situación laboral regular utilizaron esta vía. Por otro lado, la obtención del empleo por iniciativa propia es de un 30,5\% para las mujeres en situación laboral regular y de un 22,6\% si están en situación laboral irregular.

Las tres modalidades de empleo doméstico que se han analizado son:

- Trabajadora interna, que vive y duerme dentro del hogar con la familia en la que presta sus servicios. Suponen un $11,4 \%$ del colectivo. De entre este colectivo, las contratadas internas son las que gozan de peores condiciones sobre todo en lo relativo a la jornada laboral, ya que por el hecho de vivir en la casa, sus empleadores siguen contando con sus servicios excediéndose en la jornada laboral de las 40 horas semanales, horas que muy rara vez son remuneradas en metálico. Dentro del trabajo doméstico interno existe la modalidad del pago en "especie". Es decir que, a cambio de la manutención y el hospedaje, el empleador está exento de abonar la totalidad del sueldo, pudiendo disminuirlo hasta en un $45 \%$ de total.

- Trabajadora externa fija, que tiene contratada o pactada una jornada y su remuneración es generalmente una vez al mes o en función del acuerdo al que haya llegado con su empleador. El $25,8 \%$ de las trabajadoras de la muestra corresponden a esta modalidad.

- Trabajadora externa por horas, que desarrolla su trabajo y percibe una remuneración por el mismo de forma esporádica o con cierta regularidad, que no tiene porque ser periódica. Representan el $62,8 \%$ de la muestra.

Las empleadas de hogar que están en situación laboral irregular tienen un peso importante las mujeres que trabajan como externas por horas (67\%), mientras que esta cifra en las mujeres que están regularizadas desciende a un 55\%. Estos resultados son acordes, si se tiene en cuenta que determinadas trabajadoras necesitan cierta flexibilidad laboral, y es más sencillo encontrar un trabajo por horas que esa flexibilidad en las otras modalidades donde en general se demandan jornadas mayores (y dedicación exclusiva o casi exclusiva). 
En cuanto al número de casas en los que estas empleadas desarrollan su trabajo, es similar entre las que están en situación laboral regular e irregular.

Al analizar las diferencias en función de la nacionalidad, dentro de las mujeres que están en situación irregular, el 58,8\% de las que trabajan como internas tienen nacionalidad extranjera. Las españolas que trabajan como empleadas del hogar de manera irregular suelen estar buscando una flexibilidad en el horario laboral y un complemento de su renta familiar. Un dato relevante con respecto a este aspecto es que el $71 \%$ de las mujeres que trabajan dentro de la modalidad de externas por horas son españolas. En el caso de las mujeres que se encuentran en situación laboral regular, el porcentaje de mujeres nacionales que trabajan como internas sigue siendo inferior que el de las inmigrantes $(45,9 \%$ frente a un $54,1 \%)$.

Autores como Young (1990) consideran el servicio doméstico un ejemplo claro de explotación, a través del cual se produce una transferencia de los resultados del trabajo de un grupo en beneficio de otro, lo que comporta distribuciones desiguales de recursos y de riqueza. En este sentido, quien emplea adquiere algo más que el producto del trabajo de la empleada; compra a la persona en su totalidad, sobre todo cuando se trata de la modalidad del servicio doméstico interno (Anderson, 2000).

En el grupo de las trabajadoras que se dedican al servicio doméstico las que se encuentran en una situación laboral regular, el $49 \%$ tiene un contrato escrito y un $21 \%$ dicen tener un acuerdo verbal con su empleador. Entre las empleadas de hogar en situación laboral irregular, tan solo un $13 \%$ posee contrato escrito.

Entre las empleadas de hogar que se encuentran en situación laboral no regular, el $81 \%$ no cotizan y un $19 \%$ lo hacen, pero por menos horas de las que realmente trabajan.

En el caso de las trabajadoras en situación laboral regular, que son las que cotizan a la Seguridad Social, el 52,5\% cotiza el empleador y en un 26,7\% la cotización se realiza de manera proporcional entre empleador y trabajadora. Tan sólo el 20,8\% restante realizan ellas mismas la cotización.

Entre las mujeres en situación laboral irregular, donde como hemos visto la mayoría no están dadas de alta en la Seguridad Social, el principal motivo para no cotizar es que no les interesa o no les compensa cotizar (50,8\%), debido a que los salarios que perciben en general son bajos. Además hay un $16,4 \%$ que reconoce que el hecho por el cual no cotizan a la Seguridad Social se debe al desconocimiento de si deben cotizar o no. En las mujeres de nacionalidad extranjera, esta cifra es de un $22,6 \%$.

En el estudio de Cuanter (2001), basado en la aplicación de una encuesta específica a empleadas de hogar, se detecta que sólo el 17,4\% de éstas están dadas de alta en el R.E. de Empleados de Hogar y que un $46,8 \%$ carece de alta teniendo obligación a ello.

Para el año 2004, el 91,7\% de las altas de extranjeros en el Régimen Especial de Empleados de Hogar, corresponden a mujeres. Y son principalmente las nacionalidades latinoamericanas las que ocupan estos contratos laborales (Villares y col., 2005). 
La Seguridad Social tiene una gran repercusión en todos los sectores de la sociedad. Hace que las trabajadoras tengan acceso a la asistencia médica, cuenten con protección contra la pérdida de empleo y les proporcione ingresos durante sus años de vejez, por lo que como acabamos de señalar a estas trabajadoras la Seguridad Social no las ayuda a mantener relaciones laborales estables, y a contribuir a la cohesión social, ni al crecimiento y desarrollo general del país.

En cuanto a los salarios, aproximadamente en ambos casos el 18\% (situación laboral regular e irregular) cobran menos de 300 euros mensuales. Entre las mujeres en situación laboral irregular un 59,4\% recibe al mes unos ingresos de 300 a 600 euros, reduciéndose este porcentaje en el caso de las mujeres regulares a un $48,6 \%$. Por otro lado, un $22,8 \%$ de las mujeres regularizadas percibe de 600 a 1000 euros al mes, mientras que el porcentaje de las irregulares que reciben esta cuantía se reduce al $16,2 \%$.

En lo que se refiere al horario, las mujeres que están en situación laboral regular trabajan semanalmente una media de dos horas menos que las que están en situación laboral irregular ( 27 horas semanales en media frente a 29 horas semanales).

Las tareas que más frecuentemente se realizan en el servicio doméstico son: limpieza de la casa, hacer la compra, cocinar, planchar, el cuidado de niños/as y personas enfermas, discapacitadas o personas mayores.

En cuanto a las tareas realizadas por las mujeres tanto en situación laboral irregular como regular, existen diferencias por edades poco significativas, ya que tanto las mujeres jóvenes como las de edad más avanzada realizan habitualmente el cuidado de la casa y de la ropa.

\section{Búsqueda de los perfiles de las mujeres empleadas de hogar, basada en información multivariante}

La cuantificación de las variables categóricas, con el método análisis de homogeneidad, nos ha permitido determinar los perfiles de las mujeres empleadas en el servicio doméstico y encontrar grupos de mujeres con perfiles similares y detectar las variables más relevantes en la definición de los diferentes perfiles.

Para conocer las variables con mayor influencia en la clasificación, analizamos la tabla 3 que recoge los índices de discriminación; es decir, los ítems que más han contribuido a la formación de los grupos que presentan perfiles bien diferenciados. 
Tabla 3. Medidas de discriminación para clarificar la interpretación sólo se escriben los valores mayores de 0.30

\begin{tabular}{|l|c|c|}
\hline & \multicolumn{2}{|c|}{ Dimensiones } \\
\hline & $\mathbf{1}$ & $\mathbf{2}$ \\
\hline i3 Estado Civil & 0,356 & 0,329 \\
\hline i11 Donde vive actualmente & 0,574 & 0,354 \\
\hline i15.4 Otros familiares realizan las tareas del hogar & & 0,379 \\
\hline i16.4 Mis padres tienen trabajo remunerado & & 0,379 \\
\hline
\end{tabular}

En nuestro estudio aparecen cuatro conglomerados con la siguiente distribución de frecuencias:

Tabla 4. Distribución por conglomerados

\begin{tabular}{|c|c|}
\hline CONGLOMERADOS & FRECUENCIA \\
\hline 1 & 237 \\
\hline 2 & 158 \\
\hline 3 & 344 \\
\hline 4 & 139 \\
\hline
\end{tabular}

Para caracterizar los conglomerados vamos a considerar las variables más significativas: situación laboral, nacionalidad, edad y estado civil.

\section{Situación Laboral}

La tabla 5 pone de nuevo de manifiesto la situación de irregularidad que predomina en el sector doméstico. Podemos observar como los conglomerados 2, 3 y 4 son irregulares (el 62\% de las empleadas de hogar están en situación laboral no regular). En el conglomerado 1 se sitúan las mujeres en situación regular dedicadas a esta actividad.

Tabla 5. Distribución por conglomerado según situación laboral

\begin{tabular}{|c|c|c|c|}
\hline & \multicolumn{2}{|c|}{ SITUACIÓN LABORAL } & \multirow[b]{2}{*}{ TOTAL } \\
\hline & Regular & Irregular & \\
\hline CONGLOMERADO 1 & $\begin{array}{r}138 \\
\quad \mathbf{5 8 , 2} \%\end{array}$ & $41,8 \%$ & $\begin{array}{ll}237 & \\
& 100 \%\end{array}$ \\
\hline CONGLOMERADO 2 & $\begin{array}{l}35 \\
22,2 \%\end{array}$ & $77,8 \%$ & $\begin{array}{ll}158 & \\
& 100 \%\end{array}$ \\
\hline
\end{tabular}




\begin{tabular}{|r|rr|lr|rr|}
\hline CONGLOMERADO 3 & \multicolumn{1}{|l|}{118} & 226 & & 344 & \\
& & $34,3 \%$ & & $\mathbf{6 5 , 7 \%}$ & $100 \%$ \\
\hline CONGLOMERADO 4 & 43 & & 96 & & 139 \\
& & $30,9 \%$ & & $\mathbf{6 9 , 1 \%}$ & & $100 \%$ \\
\hline TOTAL & 334 & & 544 & & 878 & \\
& & $38 \%$ & & $62 \%$ & & $100 \%$ \\
\hline
\end{tabular}

\section{Nacionalidad}

Analizando los resultados obtenidos según la nacionalidad, es de destacar que dentro de esta rama de actividad la participación de las mujeres de nacionalidad extranjera es superior al papel que juegan en otros sectores de la economía.

El cuadro siguiente nos permite afirmar que los conglomerados 1 (con un 97\%), 3 (con un $81,1 \%$ ) y 4 (con un 74,1\%) están constituidos principalmente por empleadas del servicio doméstico españolas, situándose en el conglomerado 2 con un $88 \%$ las inmigrantes. El 73,3\% de éstas son procedentes de Latinoamérica, hecho que atribuimos a la facilidad para desenvolverse en nuestro país por el idioma.

Tabla 6. Distribución por conglomerado según nacionalidad

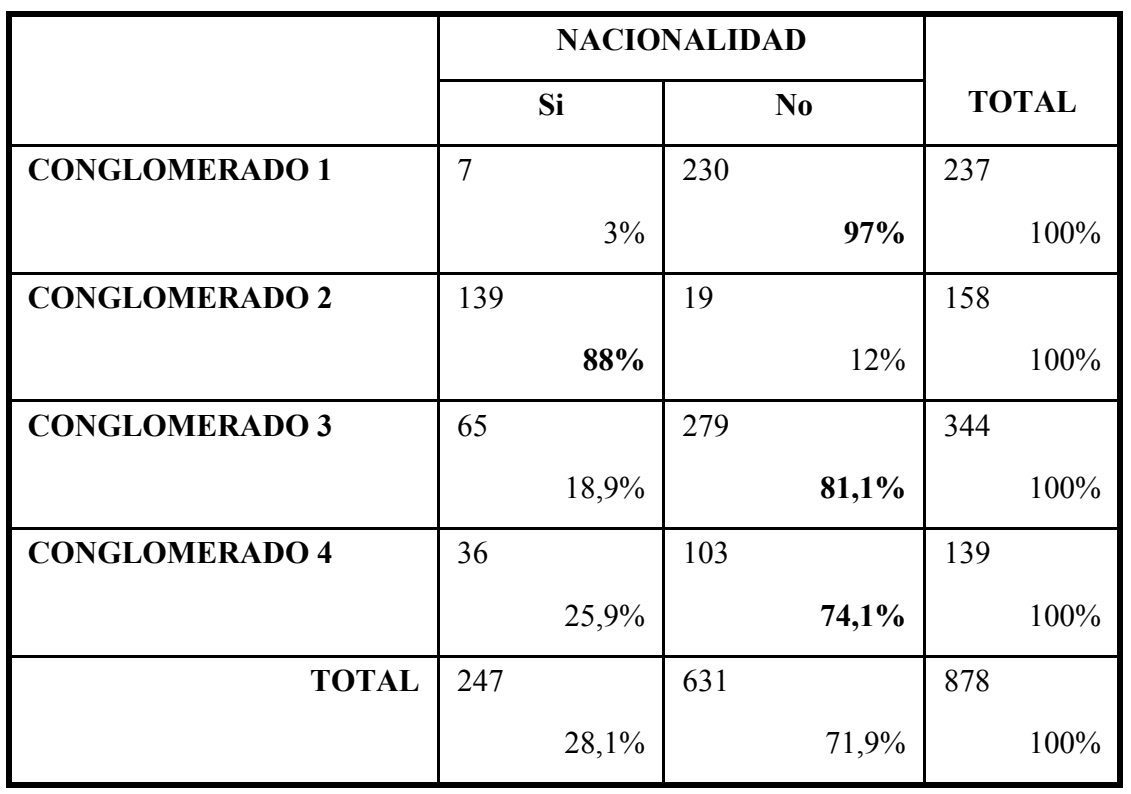




\section{Estado Civil}

El estado civil ha sido apuntado tradicionalmente como uno de los principales factores que influyen en los motivos y condiciones para que las empleadas de hogar realicen su actividad en un marco irregular. Las necesidades y la apreciación que éstas tienen del trabajo difieren entre las mujeres casadas, solteras, separadas/divorciadas o viudas. De manera general, la participación laboral de una mujer casada es vista dentro del propio hogar como una fuente secundaria de ingresos, sujeta a una serie de limitaciones en cuanto a horarios y condiciones laborales, que no son tenidas en cuenta por las mujeres solteras cuando analizan su participación en el mercado de trabajo.

Son diversas las circunstancias que pueden condicionar la participación laboral de mujeres separadas/divorciadas o viudas. Una nueva vida que les puede inducir a buscar fuentes de estabilidad. Por otro lado, la participación de estas mujeres en el sector doméstico puede ser un importante complemento a otras fuentes de ingreso (pensiones o manutención), que pueden ser incompatibles con una participación en el mercado de trabajo regular.

La tabla siguiente presenta la distribución por conglomerado según el estado civil. En los conglomerados 1 y 3, existe una importante concentración de mujeres casadas (el 86,5\% y 73,5\% respectivamente). En el conglomerado 2, constituido mayoritariamente por empleadas de hogar inmigrantes, la repartición entre mujeres casadas y solteras es un $48,8 \%$ de mujeres casadas y un $27,8 \%$ de solteras. El grupo 4 está constituido por la mayor parte de mujeres solteras de la muestra (un 91,4\%).

Tabla 7. Distribución por conglomerado según estado civil

\begin{tabular}{|c|c|c|c|c|c|}
\hline & SOLTERA & CASADA & DIVORCIADA & VIUDA & TOTAL \\
\hline CONG 1 & $4,2 \%$ & $\begin{array}{ll}205 & \\
& \mathbf{8 6 , 5 \%}\end{array}$ & $5,1 \%$ & $\begin{array}{l}10 \\
4,2 \%\end{array}$ & $\begin{array}{l}237 \\
100 \%\end{array}$ \\
\hline CONG 2 & $27,8 \%$ & $48,8 \%$ & $17,1 \%$ & $\begin{array}{r}10 \\
\quad 6,3 \% \\
\end{array}$ & $\begin{array}{l}158 \\
100 \% \\
\end{array}$ \\
\hline CONG 3 & $10,8 \%$ & $\begin{array}{ll}253 & \\
& \mathbf{7 3 , 5 \%} \\
\end{array}$ & $10,5 \%$ & $\begin{array}{ll}18 & \\
& 5,2 \% \\
\end{array}$ & $\begin{array}{r}344 \\
100 \% \\
\end{array}$ \\
\hline CONG 4 & $91,4 \%$ & 10 & $1,4 \%$ & $\begin{array}{ll}0 & \\
& 0,0 \% \\
\end{array}$ & $\begin{array}{r}139 \\
100 \% \\
\end{array}$ \\
\hline TOTAL & 218 & 545 & 77 & 38 & 878 \\
\hline
\end{tabular}

Los cuatro conglomerados definidos en el análisis se caracterizan por:

Conglomerado 1: Empleadas de hogar españolas casadas en situación laboral regular.

Este primer grupo, se corresponde con mujeres españolas maduras, con una edad entre 30 y 45 años, casadas, con una media de uno o dos hijos, que desarrollan su actividad dentro de este sector en el subgrupo que hemos denominado "limpieza de oficinas". El 67,5\% de las mujeres de este grupo tienen estudios primarios y apenas 
hay mujeres que carezcan de formación académica. Aunque en su mayoría tienen unos ingresos medios mensuales inferiores a 600 euros, es destacable el hecho de que el $28,7 \%$ de las trabajadoras de este grupo perciben mensualmente unos ingresos de 600 a 1500 euros. Tienen una presencia significativa en los municipios rurales intermedios.

Conglomerado 2: Empleadas de hogar de nacionalidad extranjera.

Es un perfil cada vez más frecuente en la última década, debido a la llegada a nuestro país de mujeres inmigrantes de diferentes países.

Este grupo está formado por mujeres de nacionalidad extranjera, en su mayor parte de Latinoamérica (que son las que predominan en nuestra muestra; no obstante las mujeres de otras nacionalidades tienen motivaciones similares, de ahí que pueda hablarse de un perfil de mujeres de nacionalidad extranjera, al margen de su origen), que tienen una edad en un intervalo entre 30 y 45 años y un nivel de estudios primarios o sin estudios en su mayoría.

Entre las mujeres que forman este grupo, el porcentaje de casadas $(48,8 \%)$ es superior a las solteras $(27,8 \%)$. Su nivel de ingresos es de 300 a 600 euros al mes.

En las zonas de ruralidad urbana y laxa es donde estas mujeres desarrollan principalmente su actividad.

Conglomerado 3: Empleadas de hogar españolas casadas en situación laboral irregular.

Este grupo se corresponde con un perfil más tradicional, está formado por mujeres de mediana edad que buscan en el mercado laboral una forma de aumentar los ingresos de la familia.

Está formado por mujeres de nacionalidad española, con una edad media que se sitúa entre los 30 y 55 años. La mayor parte de ellas están casadas y tienen hijos. En cuanto a su nivel formativo tienen un nivel de estudios primarios. La renta aportada por la mujer suele ser un complemento a la renta del hogar, ya que en el $60,3 \%$ de los casos los ingresos obtenidos por su trabajo son de 300 a 600 euros al mes. Conglomerado 4: Empleadas de hogar españolas solteras en situación irregular.

Este tercer grupo está compuesto por mujeres españolas, jóvenes, con edades comprendidas entre 20 y 29 años, sin hijos y tal como se ha dicho anteriormente en conjunto presentan un nivel académico de estudios primarios/secundarios. Su nivel de ingresos no supera los 600 euros al mes.

El Instituto de la Mujer (Cabo y col., 2005) afirma que el servicio doméstico es la actividad donde se concentra la mayor irregularidad laboral femenina, con un $30 \%$ del empleo sumergido; seguido de otras ocupaciones como los servicios a empresas, con el $16 \%$ del total; la hostelería y el pequeño comercio con un $14 \%$ y un $13 \%$, respectivamente; y la educación con un $9 \%$. 


\section{Demandas de las empleadas de hogar en situación laboral irregular para acceder al mercado de trabajo regular}

El 86,4\% de las trabajadoras salmantinas empleadas en el servicio doméstico que se encuentran en situación laboral irregular, han señalado que si pudiesen elegir prefieren tener un empleo en condiciones regulares, con contrato, cotizando a la Seguridad Social y con un Régimen de empleadas de hogar equiparable al Régimen General.

Por perfiles, entre las demandas que el grupo de mujeres casadas de nacionalidad española realizan destaca el tener acceso a las prestaciones sociales $(85,5 \%)$ y que la Administración asuma el cuidado de personas dependientes, especialmente el cuidado de las personas mayores y niños con ayudas de guarderías y residencias $(80,6 \%)$. En este bloque el grupo de mujeres de mayor edad demandan flexibilidad de horarios alegando que tienen personas enfermas y ancianos en casa y no pueden dejarlos solos, pudiendo así conciliar mejor su vida familiar y su vida laboral.

El grupo de mujeres autóctonas solteras lo que demandan en su mayoría es obtener apoyo, asesoramiento y orientación en la búsqueda de empleo $(90,7 \%)$. Se trata de mujeres jóvenes que han alcanzado un nivel educativo medio-bajo y han decidido concluir sus estudios académicos e incorporarse al mercado laboral en este sector que no requiere ningún tipo de cualificación profesional, pero que aspiran a trabajar en sectores mejor pagados.

Y por último el grupo de las mujeres inmigrantes de distintas nacionalidades, latinoamericanas en su mayoría, demandan sueldos mejor pagados y tener acceso a las prestaciones sociales $(89,5 \%)$. Es un perfil condicionado esencialmente por la necesidad de obtener ingresos para sí y sus familias y en muchos casos para poder conseguir legalizar su situación en el país.

En definitiva las demandas de estas mujeres tienden hacia la consecución de una situación laboral regular y mejoras en sus condiciones laborales con sueldos que le permitan vivir mejor.

\section{Medidas para mejorar el acceso al empleo regular}

Las medidas que el grupo de mujeres casadas que están en una situación laboral irregular consideran que les ayudarían a mejorar el acceso a un trabajo regular son principalmente una reducción de las cotizaciones sociales $(80,8 \%)$ y ofrecer servicios de apoyo y orientación a las mujeres en la búsqueda de un trabajo $(89,4 \%)$, coincidiendo en darle una importancia significativa a este aspecto con el grupo de las mujeres de nacionalidad extranjera $(93,8 \%)$.

El perfil de las mujeres solteras solicita cursos con contenido práctico que les prepare para el mundo laboral: idiomas, cocina, albañilería, puericultura, peluquería, jardinería, informática, contabilidad, etc. La mayoría coinciden en que los contenidos de los cursos a los que han asistido son demasiado alejados de lo que luego 
realmente necesitan al incorporarse a un puesto de trabajo y piden que se facilite el acceso a esos cursos de formación.

La mayoría de estas mujeres que trabajan en situación laboral irregular coincide en que una disminución de los impuestos para establecerse por su cuenta y aumentar la inspección laboral e incrementar las responsabilidades penales, contribuiría a resolver muchos de los casos de irregularidad.

\section{Conclusiones}

Nuestra investigación es la primera que aborda el estudio para la mujer empleada de hogar salmantina y los resultados apuntan a una provincia caracterizada por un elevado peso del servicio doméstico, presentando una elevada concentración en los municipios no rurales (urbanos).

El estudio que se realizó a través de diez entrevistas en profundidad y 878 encuestas personales, realizadas en 68 municipios salmantinos, compara las características socioeconómicas de las trabajadoras en el servicio doméstico en situación laboral regular e irregular, e identifica cuatro conglomerados bien diferenciados, dos constituidos por mujeres de nacionalidad española en situación laboral no regular: casadas y solteras. Otro, constituido por mujeres inmigrantes que buscan un complemento monetario para sus gastos personales. Y un último grupo formado por las mujeres empleadas de hogar regularizadas.

Las mujeres empleadas de hogar salmantinas presentan unos niveles educativos primarios o sin estudios, tratándose en su mayoría de mujeres maduras, casadas.

La edad media de las trabajadoras es de 40 ó más años. Las variables, edad y cualificación académica se encuentran por tanto muy relacionadas. Éste es sin duda el perfil tradicional del servicio doméstico. El estudio aporta un resultado novedoso a este respecto desagregando un único perfil en los cuatro conglomerados resultado del análisis multivariante de Correspondencias Múltiples.

El $60,4 \%$ de las trabajadoras en el servicio doméstico irregular tiene unos ingresos mensuales inferiores a 300 euros. La retribución media de las trabajadoras en situación laboral regular es superior a la de las mujeres en situación laboral irregular.

Las mujeres en situación laboral irregular salmantinas empleadas de hogar solicitan prioritariamente el acceso a prestaciones sociales y una ayuda por parte de la Administración en la atención de niños y ancianos, que les permita conciliar su vida familiar y su vida laboral.

Es un sector donde las cotizaciones a la Seguridad Social son muy bajas y las trabajadoras no están dadas de alta. 


\section{Discusión}

Existe un porcentaje significativo en la muestra (28\%) de mujeres de nacionalidad extranjera, hecho que atribuimos a la fuerte inmigración durante los últimos años en nuestro país, señalando muchas de ellas que desarrollan su actividad en dicho sector por no tener papeles y no tener por tanto otra alternativa. El estudio llevado a cabo por Escrivá (2000) “¿Empleadas de por vida? Peruanas en el servicio doméstico de Barcelona", coincide en que las inmigrantes entrevistadas aparecen desencantadas al poco tiempo de llegar por las condiciones tan adversas para ellas, como son la escasez de trabajos bien pagados y el alto costo de la vida como de los alquileres, desarrollando su actividad laboral básicamente como empleadas de hogar. Sin embargo, a diferencia de su trabajo que encuentra cuatro situaciones dentro de las mujeres de nacionalidad extranjera ya asentadas (por un lado las que tienen necesidades económicas familiares y ven necesaria su perpetuación como empleadas de hogar internas y externas, en las que se produce un cambio más o menos intenso de movimientos de empleo dentro del sector de limpiezas, las que dejan el servicio doméstico remunerado para ejercerlo en su hogar y en las que se produce un paso hacia sectores laborales más revalorizados), para el caso de Salamanca hemos diferenciado por un lado las que permanecen en el sector del servicio doméstico por las necesidades económicas que tienen en sus hogares, las que abandonan para pasar a trabajar dentro del sector limpiezas de oficinas y portales y un tercer grupo que se dedican al cuidado de personas dependientes, cuidadoras de ancianos y discapacitados, coincidiendo con Martínez (2009) en que la principal vía de la asistencia dirigida a personas mayores es el servicio doméstico.

En cuanto a la modalidad de empleo doméstico, para el caso de Salamanca el porcentaje de trabajadoras empleadas como internas (11\%) es superior a otros estudios oscilando la media en torno a un 7\% como el realizado por Herranz (1997) "Mujeres dominicanas en el servicio doméstico de Pozuelo-Aravaca" el cual expone como la mano de obra en el servicio doméstico interno es mayoritario en las mujeres de origen extranjero las cuales, en su mayoría trabajan como externas, o por horas, y siendo además el servicio doméstico el principal sector en el que se incorpora la mujer inmigrante en Madrid, hecho coincidente con el caso de Salamanca, pero difiere en la modalidad del empleo: para el caso de Pozuelo el 88,1\% trabajaba todo el día, es decir en el servicio doméstico interno, predominando en nuestra muestra objeto de estudio las trabajadoras externas por horas $(62,8 \%)$. La modalidad de externas fijas otras investigaciones la ponen como predominante (Cuanter, 2001), lo cual viene explicado por la mayor satisfacción que el grupo de las empleadas de hogar solteras y casadas no regularizadas expresan con respecto al horario de trabajo y vacaciones. Sin embargo muestran una gran insatisfacción con respecto a las pagas extraordinarias y salario y presentan unas rentas de trabajo inferiores a 600 euros al mes.

Rodríguez (1989) expone que si relacionamos el número de horas trabajadas fuera del hogar con las personas que ayudan a las mujeres dentro de casa, observamos que más del $90 \%$ de las mujeres que tienen empleada de hogar, trabajan la jornada 
completa. Como era de esperar, al disminuir el número de horas que las mujeres trabajan fuera de casa, disminuye también la ayuda externa -empleadas del hogar y madres-.

El servicio doméstico es la actividad donde se concentra la mayor irregularidad laboral femenina, un 36\% del empleo irregular total, seguido de la hostelería con un $25 \%$, y el pequeño comercio. Estos resultados son concordantes con los dados por Cabo y col. (2005), en su trabajo "La presencia de las mujeres en el empleo irregular" que llevaron a cabo para toda España. Quiere decir esto que en las estimaciones sacadas del estudio, Salamanca tiene un comportamiento coincidente con el encontrado a nivel de toda España.

En países como Grecia se ha producido también desde 1990 la llegada de mujeres procedentes de los Balcanes y países ex socialistas de Europa central y oriental dedicándose en su mayoría a la actividad del servicio doméstico, cuidado de niños y personas mayores, siendo muy precarias tanto las condiciones laborales como las cotizaciones con respecto a la Seguridad Social (Vassilikou, 2007).

Las condiciones de trabajo descritas unido a la falta de reconocimiento social del trabajo doméstico remunerado incluso desde la propia percepción de las trabajadoras son elementos clave para definir al sector en una situación de precariedad laboral.

\section{Bibliografía}

Anderson, B. J. (2000). Doing the dirty work?. The global politics of domestic labour. London: Zed Books.

Cabo Serrano, G.; González Martín, A.; Roces, P. y Muñoz Polo, S. (2005). La presencia de las Mujeres en el Empleo Irregular. Centro de Estudios Económicos Tomillo, Instituto de la Mujer, Madrid.

Castillo Alonso, J.J. (2005). Contra los estragos de la subcontratación: trabajo decente. Sociología del Trabajo. Núm. 54, 3-37.

Colectivo Ioé. (1990). El servicio doméstico en España. Entre el trabajo invisible y la economía sumergida. JOC-E: Madrid.

Colectivo Ioé. (1996). Procesos de Inserción y Exclusión Social de las Mujeres Inmigrantes no Comunitarias. Instituto de la Mujer, Madrid.

Cuanter S.A. (2001). Estudio Sociosanitario de las Empleadas de Hogar. Instituto de la Mujer. Madrid.

Destremau, B. y Lautier, B. (2002). Femmes en domesticité. Les domestiques du sud, au nord et au sud. Revue Tiers Monde. Vol. 43 Núm. 170, 249-264. 
Escrivá Chorda, M.A.. (2000). ¿Empleadas de por vida? Peruanas en el servicio doméstico de Barcelona. Papers Revista de Sociología. Vol. 60, 2000, 327-342.

Galindo Villardón, P. (1986). Una alternativa de representación simultánea: HJBiplot. Qüestiió. Vol.10 Núm.1, 13-23.

García Sanz, B. (1998). La Sociedad Rural de Castilla y León Ante el Próximo Siglo. Junta de Castilla y León (pp 324). Valladolid.

Giddens, A. (1989). Sociología. Madrid, Alianza Universidad Textos.

Gifi, A. (1990). Nonlinear Multivariate Analysis. Chichester, Wiley.

Gómez, E. (2008). Sólo una de cada 3 empleadas del hogar cotiza a la Seguridad Social. Tribuna Salamanca, 6 Nov, 12.

González Martínez, M.P. (1982). Notas sobre la condición de la mujer trabajadora en España durante los tres primeros decenios del siglo XX. En Actas de las Primeras Jornadas de Investigación Interdisciplinaria. Nuevas perspectivas sobre la mujer. (Vol.2, pp 97-104) Madrid: Universidad Autónoma.

Herranz Gómez, Y. (1997). Mujeres dominicanas en el servicio doméstico de Pozuelo-Aravaca. Cuadernos de Relaciones Laborales. Vol. 10 Núm. 11, 75-101.

Izquierdo Escribano, A. López de Lera, D. y Martínez Buján R. (2003). The Favourites of the Twenty-First Century: Latin American Immigration in Spain. Studi Emigrazione. Vol. 149, 98-124.

Luján Alcaraz, J. (2000). El Régimen Especial de la Seguridad Social de los Empleados de Hogar. Elcano, Aranzadi.

Martínez Buján, R. (2009). ¿Y qué pasa con mi cuidadora? Inmigración, servicio doméstico y privatización de los cuidados a las personas dependiente. Zerbitzuan. $N^{o} 45,99-109$.

Mirás Araujo, J. (2005). Rasgos básicos y transformaciones en el Servicio Doméstico en una ciudad periférica. A Coruña, 1900-1960. Cuadernos de Historia Contemporánea. Vol. 27, 197-221.

Nielfa Cristóbal, G. (1993). Mujer y trabajo. Cuadernos del Mundo Actual. Vol. 35, 5-31.

Quesada Segura, R. (1991). El contrato del servicio doméstico. La Ley. 
Rodríguez Moya, J. M. (1989). La Mujer de Torrejón de Ardoz ante el Mercado de Trabajo. Anales de Geografía de la Universidad Complutense. Vol. 9, 215-230.

Ruiz Quintanilla A. y Wilpert, B. (1988). The meaning of working. Scientific status of a concept. En DE KEYSER, Veronique y otros (eds.) (1988): The meaning of work and technological options, John Wiley \& Sons Ltd.

Sarasúa García, C. (1983). El servicio doméstico en el Madrid del XIX. Historia.Vol. 16,89, 19-26.

Vassilikou, C. (2007). Inmigrant Women in Greece. A biographical Study of Domestic Workers. Gender, rovne prilezitosti, vyzkum. Vól. 8 Núm 1, 40-45.

Villares Varela, M.; Pérez Caramés, A.; Martínez Buján, R.; Golías Pérez, M. y Fernández Suárez, B. (2005). Los nichos laborales de la Inmigración femenina en España: del desempeño de tareas reproductivas al autoempleo. Mobilités au féminin Tánger. 15-20.

Young, I. M. (1990). Justice and the Politics of Difference. Princeton: NJ: Princeton. University Press. 